\title{
Magnetoelectric Effect in Lorentz-violating Extensions of the Standard Model
}

\author{
Oleg Kharlanov ${ }^{1, \star}$ and Ivan Degtev ${ }^{2}$ \\ ${ }^{1}$ Lomonosov Moscow State University, Faculty of Physics, 1/2 Leninskie Gory, 119991 Moscow, Russia \\ ${ }^{2}$ Lebedev Physical Institute of the Russian Academy of Sciences, 53 Leninskiy Prospekt, 119991 Moscow, \\ Russia
}

\begin{abstract}
We demonstrate that an atom placed in a constant background interacting with the electronic axial-vector current exhibits a new type of parity-breaking transverse magnetoelectric polarizability. Within beyond-Standard-Model (BSM) frameworks, such as the Lorentz-violating Standard Model Extension, such constant backgrounds are considered to be condensates of Planck-scale fields, thus, precision measurements of the magnetoelectric polarizability is able to constrain non-trivial BSM physics. Indeed, we demonstrate that the contribution to this effect within the Standard Model due to weak interaction is strongly suppressed, the effect virtually being purely exotic. We calculate magnetoelectric polarizability for a simple atom and discuss its observability.
\end{abstract}

\section{Introduction}

Lorentz invariance has been an underlying principle of virtually all modern physical theories since their emergence, including quantum field theory and high-energy physics. With the advent of the string theory and other attempts to find a consistent description of quantum gravity, however, it turned out that this symmetry could be spontaneously broken on an energy scale close to the Planck energy [1]. Moreover, a possibility for dynamical Lorentz violation by radiative corrections was also pointed out in field theories, such as the Axion-Wess-Zumino (AWZ) model [2]. Finally, quite recently, Petr Hořava presented an example of a field theory, whose non-Lorentzian behavior in the highenergy regime results in power-counting renormalizability, whereas in the low-energy regime, the conventional $\mathrm{SO}(1,3)$ symmetry group is restored and the theory reproduces General Relativity [3]. These discoveries have triggered interest in precision tests of Lorentz invariance one could perform at attainable energies, as a window into beyond-Standard-Model (BSM) physics.

In order to implement the strategy of constraining BSM physics with precision tests of Lorentz invariance, the Standard Model Extension (SME) framework was proposed [4]. This framework assumes that high-energy particles present in the hypothetical fundamental theory have masses that are too large to be observable at any of the present experiments, while signatures of Lorentz violation could be found in condensates of Planck-scale fields whose nonzero values led to the corresponding symmetry breaking. Thus, SME is assumed to be a low-energy mean-field theory with a number of order parameters interacting with conventional fields featuring in the Standard Model via a set of extra

^e-mail: kharlanov@physics.msu.ru 
terms in the SME Lagrangian. Note that no additional dynamical fields are present in SME, compared with the Standard Model. Diversity of exotic terms in SME is usually limited by a number of 'natural requirements', such as energy-momentum conservation, $\mathrm{SU}(3)_{c} \times \mathrm{SU}(2)_{I} \times \mathrm{U}(1)_{Y}$ gauge invariance, and anomaly cancellation; as a result, the number of allowed Lorentz-violating terms can even be made finite (for details, see Ref. [4]).

After the electroweak symmetry breaking, SME leads to extended electrodynamics, whose Lagrangian reads

$$
\begin{gathered}
\mathcal{L}_{\mathrm{EQED}}=-\frac{1}{4} F_{\mu \nu}^{2}+\frac{1}{2} \epsilon_{\mu \nu \alpha \beta} k_{A F}^{\mu} A^{v} F^{\alpha \beta}-\frac{1}{4} k_{F}^{\mu \nu \alpha \beta} F_{\mu \nu} F_{\alpha \beta}+\bar{\psi}\left(\mathrm{i} \Gamma^{\mu}\left(\partial_{\mu}+\mathrm{i} e A_{\mu}\right)-M\right) \psi, \\
\Gamma^{\mu}=\gamma^{\mu}+c^{\nu \mu} \gamma_{v}-d^{\nu \mu} \gamma_{v} \gamma_{5}+e^{\mu}+\mathrm{i} f^{\mu} \gamma_{5}+\frac{1}{2} g^{\alpha \beta \mu} \sigma_{\alpha \beta}, \quad M=m+a^{\mu} \gamma_{\mu}-b^{\mu} \gamma_{\mu} \gamma_{5}+\frac{1}{2} H^{\mu v} \sigma_{\mu \nu},
\end{gathered}
$$

where $m$ and $e$ are the electron mass and charge, respectively, and $k_{A F}, k_{F}, a, b, \ldots, H$ are constant (pseudo)tensor couplings (condensates) with certain symmetry properties; $\gamma_{\mu}, \gamma_{5}, \sigma_{\mu \nu}$ are conventional $4 \times 4$ Dirac matrices and the electromagnetic field strength $F_{\mu \nu}=\partial_{\mu} A_{\nu}-\partial_{\nu} A_{\mu}$. Note that the same 'Lorentz-violating' terms can also describe physical situations arising within the conventional Standard Model, e.g., the axial-vector $b^{\mu}$ term can arise in intensive neutrino fluxes due to forward neutrino-electron scattering [5]. This term can also reflect the presence of a nonzero background torsion [6]. In the present paper, we study the effect of the $b^{\mu}$ term, taking

$$
\mathcal{L}=-\frac{1}{4} F_{\mu \nu}^{2}+\bar{\psi}\left(\gamma^{\mu}\left(\mathrm{i} \partial_{\mu}-e A_{\mu}+b_{\mu} \gamma_{5}\right)-m\right) \psi
$$

and consider the magnetoelectric response of a hydrogen atom, i.e., induction of an electric dipole moment of the atom by a weak external magnetic field, and vice versa. Electric and magnetic field strengths being a 3-vector and a 3-pseudovector, respectively, the corresponding polarizability $\kappa_{\mathrm{ME}}$ should be parity-violating, thus, one can expect its exotic nature, e.g., from the timelike component of the axial condensate $b^{\mu}$. Indeed, the ground state of a hydrogen atom is characterized by the only (pseudo)vector $\boldsymbol{S}$ of the electron spin, in the case of a moving atom one also has the velocity $\boldsymbol{V}$ of the nucleus, which is a polar vector. Now, to construct a pseudovector $\boldsymbol{\mu}$ of the magnetic dipole moment induced by a (polar) vector electric field $\boldsymbol{E}$ in the $b^{0}=0$ case, one can only choose $\boldsymbol{\mu} \propto[\boldsymbol{V} \times \boldsymbol{E}]$ (trilinear expressions in $\boldsymbol{S}, \boldsymbol{V}, \boldsymbol{E}$ are T-even and cannot contribute to the T-odd magnetic moment), but such a contribution is odd in the velocity and its net effect should therefore be suppressed for systems of atoms with symmetric velocity distributions. In the $b^{0} \neq 0$ case, however, another possibility, namely, $b^{0}[\boldsymbol{E} \times \boldsymbol{S}]$, opens up, which is nonzero for atoms at rest. In search of such an effect, we thus take $b^{\mu}=(b, \boldsymbol{0})$ in the rest frame of the nucleus and assume $b$ to be small. Note that similar effects, having to do with mixing of electric and magnetic observables, have been discussed recently to appear in core-collapse supernovae, Weyl semimetals, and heavy-ion collisions [7-9].

The paper is organized as follows: in Sec. 2, we introduce the setup of the problem in question and evaluate the magnetoelectric polarizability of an atom; Sec. 3 discusses Standard-Model contributions to the ME effect due to weak interaction; the concluding Sec. 4 summarizes the results obtained.

\section{Magnetoelectric effect in a hydrogen atom in the $b$ condensate}

The electron in a hydrogen atom is described by the following 1-particle Hamiltonian, which follows from (3) (we adopt units with $\hbar=c=1$ hereinafter),

$$
\hat{H}=\boldsymbol{\alpha}(\hat{\boldsymbol{p}}-e \boldsymbol{A})+e A^{0}+m \gamma^{0}+b \gamma_{5}, \quad \boldsymbol{\alpha} \equiv \gamma^{0} \gamma, \hat{\boldsymbol{p}} \equiv-\mathrm{i} \boldsymbol{\nabla} .
$$


We will work in the nonrelativistic (Pauli) approximation, in which the $2 \times 2$ Hamiltonian reads [10]

$$
\hat{h}_{b}=\frac{(\hat{\boldsymbol{p}}-e \boldsymbol{A}-b \boldsymbol{\sigma})^{2}}{2 m}+e A^{0}(\boldsymbol{x})-\frac{e}{2 m} \boldsymbol{\sigma} \boldsymbol{B}
$$

where $\boldsymbol{\sigma}$ are the Pauli matrices, $A^{\mu}(x)=\left(\varphi_{\text {nucl }}(r)-\boldsymbol{E} \cdot \boldsymbol{x}, \boldsymbol{A}\right), \varphi_{\text {nucl }}(r), \boldsymbol{E}$, and $\boldsymbol{B}=\boldsymbol{\nabla} \times \boldsymbol{A}$ being the potential of the nucleus and the external electric and magnetic fields, respectively. To evaluate the magnetic moment induced by $\boldsymbol{E}$ in the ground state of the atom

$$
\boldsymbol{\mu}(\boldsymbol{E})=-\frac{\partial\left\langle\psi_{\boldsymbol{E}, \boldsymbol{B}}\left|\hat{h}_{b}(\boldsymbol{E}, \boldsymbol{B})\right| \psi_{\boldsymbol{E}, \boldsymbol{B}}\right\rangle}{\partial \boldsymbol{B}}=-\left\langle\psi_{\boldsymbol{E}, \mathbf{0}}\left|\frac{\partial \hat{h}_{b}(\boldsymbol{E}, \boldsymbol{B})}{\partial \boldsymbol{B}}\right|_{\boldsymbol{B}=\mathbf{0}} \mid \psi_{\boldsymbol{E}, \mathbf{0}}\right\rangle,
$$

we will need the ground-state wave function $\psi_{\boldsymbol{E}, \boldsymbol{0}}$ of $\hat{h}_{b}$ in a weak electric field. The latter can be found by virtue of a unitary transformation that eliminates $b$ from the Hamiltonian (5) [10]

$$
\hat{h}_{b}(\boldsymbol{E}, \mathbf{0})=e^{\mathrm{i} b \sigma \hat{x}} \hat{h}_{0}(\boldsymbol{E}, \mathbf{0}) e^{-\mathrm{i} b \sigma \hat{x}}+O\left(b^{2}\right), \quad\left|\psi_{\boldsymbol{E}, \mathbf{0}}\right\rangle \approx e^{\mathrm{i} b \sigma \hat{x}}\left|\psi_{\boldsymbol{E}, \mathbf{0}}^{(0)}\right\rangle,
$$

where $\psi_{\boldsymbol{E}, \mathbf{0}}^{(0)}$ is the wave function in the conventional, $b=0$ case. Plugging this transformation into the definition (6) of the magnetic moment and subsequent perturbative expansion in $\boldsymbol{E} \rightarrow 0$ yield

$$
\begin{aligned}
\boldsymbol{\mu} & \approx-\mu_{\mathrm{B}}\left\langle\psi_{\boldsymbol{E}, \mathbf{0}}^{(0)}|(\hat{\boldsymbol{l}}+\boldsymbol{\sigma}+2 b[\boldsymbol{\sigma} \boldsymbol{x}])| \psi_{\boldsymbol{E}, \mathbf{0}}^{(0)}\right\rangle \\
& =\boldsymbol{\mu}_{0}-\left(2 e b \mu_{\mathrm{B}} \sum_{v \neq \text { gnd }} \frac{\langle v|\boldsymbol{E} \cdot \boldsymbol{x}| \mathrm{gnd}\rangle\langle\mathrm{gnd}|[\boldsymbol{\sigma} \boldsymbol{x}]| v\rangle}{E_{v}^{(0)}-E_{\text {gnd }}^{(0)}}+\text { c.c. }\right)+O\left(\boldsymbol{E}^{2}\right)
\end{aligned}
$$

where $\mu_{\mathrm{B}}=-e / 2 m>0$ is the Bohr magneton, $\hat{\boldsymbol{l}} \equiv \hat{\boldsymbol{x}} \times \hat{\boldsymbol{p}}$, and $\boldsymbol{\mu}_{0}$ is the magnetic moment of the ground state $|\mathrm{gnd}\rangle$ for $\boldsymbol{E}, \boldsymbol{B}, b=0$; the excited states $|v\rangle$ have the energies $E_{v}^{(0)}$ inversely proportional to the principal quantum number $n$ squared.

Note now that the above expression is featuring matrix elements of the coordinate operator $\boldsymbol{x}$, which leads to selection rules, namely, nonvanishing contributions to the sum over $v$ come from the $n \mathrm{p}_{x, y, z}$ states $(n=2,3,4, \ldots)$ having the same spin polarization as the ground state. That is, one can use the real spherical harmonics for these states, $\psi_{n p_{x, y, z}}^{(0)}(\boldsymbol{x}) \propto R_{n \mathrm{p}}(|\boldsymbol{x}|) \frac{\boldsymbol{e}_{x, y, z} \cdot \boldsymbol{x}}{|\boldsymbol{x}|}, \boldsymbol{e}_{x, y, z}$ being the three Cartesian basis vectors, thus arriving at a sum over $n$ and the angular momentum projection. Quite surprisingly, this sum can be evaluated analytically [11], leading to the magnetic momentum shift induced by the external electric field

$$
\delta \boldsymbol{\mu} \equiv \boldsymbol{\mu}(\boldsymbol{E})-\boldsymbol{\mu}_{0} \approx \frac{72 e b r_{\mathrm{B}}^{2}}{\mathrm{Ry}}[\boldsymbol{E} \times \boldsymbol{S}] \mu_{\mathrm{B}},
$$

where $r_{\mathrm{B}}=1 / m e^{2}$ is the Bohr's radius and Ry $=e^{2} / 2 r_{\mathrm{B}} \approx 13.6 \mathrm{eV}$. Note that the effect we have calculated is linear in the condensate, unlike many other effects associated with it, including the energy shifts [10]. Moreover, technically, the above expression does not include the contribution of the delocalized states with $E>0$, however, their account changes the numerical factor in (9) by no more than $5 \%$ (note that continuum states should be used with care, since their wave functions are delocalized and sensitive to the environment of the atom). Moreover, a dual effect, i.e., a nonzero electric dipole induced by the external magnetic field, also exists and exhibits the same structure of the expression $\boldsymbol{d} \propto \frac{e b r_{\mathrm{B}}^{2}}{\mathrm{Ry}}[\boldsymbol{B} \times \boldsymbol{S}] e r_{\mathrm{B}}$. Both effects are transverse, since the induced dipole moments are perpendicular to the external field, for example, the magnetoelectric polarizability tensor describing $\delta \boldsymbol{\mu}(\boldsymbol{E})$ reads

$$
\left(\kappa_{\mathrm{ME}}\right)_{i j}=\frac{72 e b r_{\mathrm{B}}^{2}}{\mathrm{Ry}} \epsilon_{i j k} S_{k}, \quad \delta \boldsymbol{\mu} \equiv \kappa_{\mathrm{ME}} \cdot \boldsymbol{E} .
$$


It is worth examining, however, whether any Standard-Model effect can produce nontrivial magnetoelectric responses similar to Eq. (9) that could mask the Lorentz-violating contributions to the effect. In the above, we have identified the source of the nontrivial magnetoelectric polarizability in the $\mathrm{P}$ parity violation. Therefore, in search for the non-exotic ME effect, one is led towards the study of weak interactions in a hydrogen atom.

\section{Magnetoelectric effect from the weak interaction of the electron and the nucleus}

Weak interactions in atomic systems have been studied since 1970s [12, 13] using precision optical resonant techniques, which has revealed a tiny but observable effect of the electron-nucleus interaction. For a spin-0 nucleus, this interaction can be written in a four-fermion fashion, leading to the following additional term in the electron Hamiltonian

$$
\hat{H} \supset-G \gamma_{5} \delta^{3}(\boldsymbol{x})
$$

where the $G$ factor is proportional to the Fermi constant and the weak charge of the nucleus [14]. Formally, the above term looks like an interaction of the electron with the $b$ condensate localized at the origin. In the nonrelativistic regime, such a term results in a correction to the Pauli Hamiltonian, namely, now, instead of Eq. (5), one has

$$
\hat{h}_{G}=\frac{(\hat{\boldsymbol{p}}-e \boldsymbol{A})^{2}}{2 m}+e A^{0}(\boldsymbol{x})+\frac{G}{2 m}\left\{\boldsymbol{\sigma} \cdot \hat{\boldsymbol{p}}, \delta^{3}(\hat{\boldsymbol{x}})\right\},
$$

where braces denote the anticommutator. Let us now analyze whether this, competing mechanism of $\mathrm{P}$ violation in the Standard Model is able to generate a nontrivial magnetoelectric response $\delta \boldsymbol{\mu}$ to a weak external field $\boldsymbol{E}$. Now we cannot use the transformation (7) to get rid of the parity-violating $G$ term, thus, we treat both the $G$ term and the external electric field term $-e \boldsymbol{E} \cdot \boldsymbol{x}$ as perturbations. By extracting the terms that are linear in both $\boldsymbol{E}$ and $G$ in the magnetic moment (6), we arrive at

$$
\delta \boldsymbol{\mu} \approx-\frac{e G \mu_{\mathrm{B}}}{2 m} \sum_{\nu, \lambda \neq \text { gnd }} \frac{\left\langle\operatorname{gnd}\left|\left\{\boldsymbol{\sigma} \cdot \hat{\boldsymbol{p}}, \delta^{3}(\boldsymbol{x})\right\}\right| v\right\rangle\langle v|(\boldsymbol{l}+\boldsymbol{\sigma})| \lambda\rangle\langle\lambda|\boldsymbol{E} \cdot \boldsymbol{x}| \mathrm{gnd}\rangle}{\left(E_{\nu}^{(0)}-E_{\mathrm{gnd}}^{(0)}\right)\left(E_{\lambda}^{(0)}-E_{\mathrm{gnd}}^{(0)}\right)}+\text { c.c. }
$$

The matrix element of $\boldsymbol{x}$ selects only the p states among all possible $\lambda$ 's; action of $\boldsymbol{l}+\boldsymbol{\sigma}$ does not change the total momentum, so $v$ states should also be p states. Now, one can express these states in terms of real spherical harmonics, and a careful analysis of the structure of the scalar products reveals that the contributions coming from the $\boldsymbol{l}$ and the $\sigma$ operators in the central term in the numerator cancel each other, leading to

$$
\delta \boldsymbol{\mu}=0 .
$$

In principle, the above result is a consequence of the localization of the weak interaction term at the origin (because of the Dirac delta function), where the wave functions of $\mathrm{p}$ states vanish. Also, the anomalous magnetic moment of the electron transforms $\boldsymbol{l}+\boldsymbol{\sigma}$ into an imbalanced combination with nonequal coefficients and the cancelation (14) does not take place. In any case, we conclude that the suppression of the magnetoelectric effect in the Standard Model is quite strong.

\section{Conclusion}

The effect we have studied in the present paper has a number of features that distinguish it favorably as a basis for precision tests of Lorentz invariance. Firstly, the corresponding effects in the Standard 
Model cancel quite surprisingly in the leading order (to the best of our knowledge, this does not happen due to any obvious symmetry of the system). Secondly, the effect is linear in the small Lorentzviolating coupling, unlike other effects the latter one generates, which are at least quadratic. On the other hand, the magnetoelectric polarizability is nonzero for spin-polarized atoms, so certain polarization techniques should be used to avoid averaging it to zero. For the latter reason, the Lorentz-induced magnetoelectric effect might show up in magnetic materials with nonzero mean magnetization.

\section{References}

[1] V. A. Kostelecký and S. Samuel, Phys. Rev. D 40, 1886 (1989).

[2] A. A. Andrianov, R. Soldati, and L. Sorbo, Phys. Rev. D 59, 025002 (1999).

[3] P. Hořava, Phys. Rev. D 79, 084008 (2009).

[4] D. Colladay and V. A. Kostelecký, Phys. Rev. D 58, 116002 (1998).

[5] A. I. Studenikin, Phys. Atom. Nucl. 67, 993 (2004).

[6] I. L. Shapiro, Phys. Rept. 357, 113 (2002).

[7] A. F. Bubnov, N. V. Gubina, and V. Ch. Zhukovsky, Phys. Rev. D 96, 016011 (2017).

[8] M. Dvornikov, JCAP 05(2015), 037 (2015).

[9] K. Fukushima, D. E. Kharzeev, and H. J. Warringa, Phys. Rev. D 78, 074033 (2008).

[10] O .G. Kharlanov and V. Ch. Zhukovsky, J. Math. Phys. 48, 092503 (2007).

[11] H. Bethe and E. E. Salpeter, Quantum Mechanics of One- and Two-Electron Atoms (SpringerVerlag, Berlin, 1957).

[12] M. A. Bouchiat and C. C. Bouchiat, Phys. Lett. B 48, 111 (1974).

[13] V. N. Novikov and I. B. Khriplovich, JETP Lett. 22(3), 74 (1975).

[14] F. Curtis-Michel, Phys. Rev. 138B, 408 (1965). 